\title{
Endoscopic ultrasound of splenic artery pseudoaneurysm
}
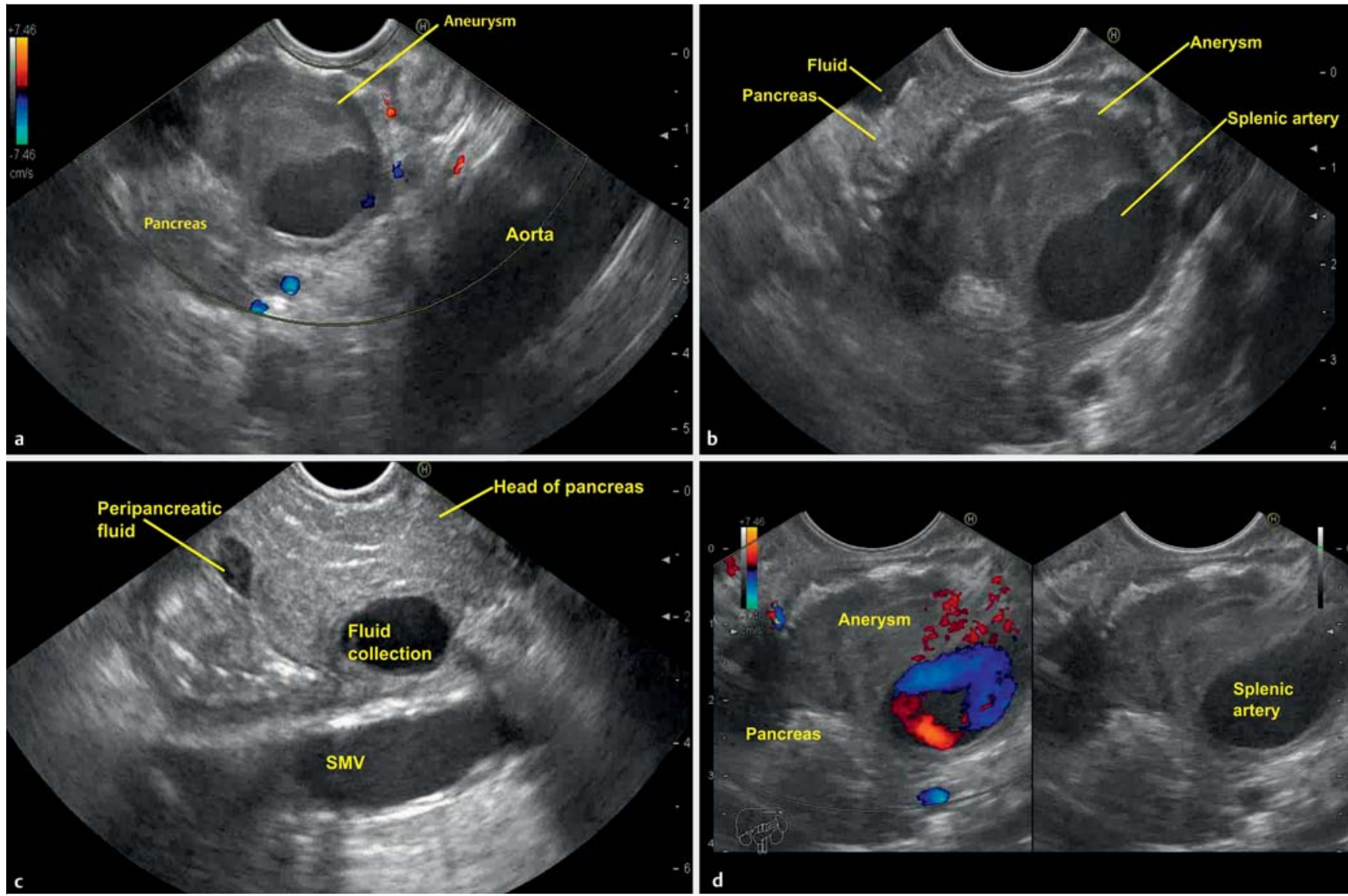

- Fig. 1 Linear endoscopic ultrasonography. a A $25 \times 22 \mathrm{~mm}$ cystic-appearing lesion was visible in the body of the pancreas. $\mathbf{b}$ The cystic lesion had a thick outer hypoechoic wall, with an inner anechoic area, giving it a characteristic appearance of a "donut." c Endoscopic ultrasound showed edematous pancreatic parenchyma with peripancreatic fluid collection consistent with an acute attack of pancreatitis. $\mathbf{d}$ On color-flow and Doppler ultrasound imaging, vascular flow was seen in the central anechoic area. SMV, superior mesenteric vein.

A 48-year-old man was referred from an outside hospital with a recent episode of acute pancreatitis and hematemesis for the evaluation of a cystic lesion in the body of the pancreas, which was noted on computed tomography (CT) of the abdomen.

Upper gastrointestinal endoscopy was normal. Linear endoscopic ultrasonography (EUS) performed from the stomach revealed a $25 \times 22 \mathrm{~mm}$ cystic-appearing lesion in the body of the pancreas. The lesion had a thick outer hypoechoic wall $(12 \mathrm{~mm})$, with a central anechoic area that had the appearance of a "donut" ( $\vee$ Fig. 1 a, b, $\triangleright$ Video 1). The surrounding pancreatic parenchyma was edematous with peripancreatic fluid collection

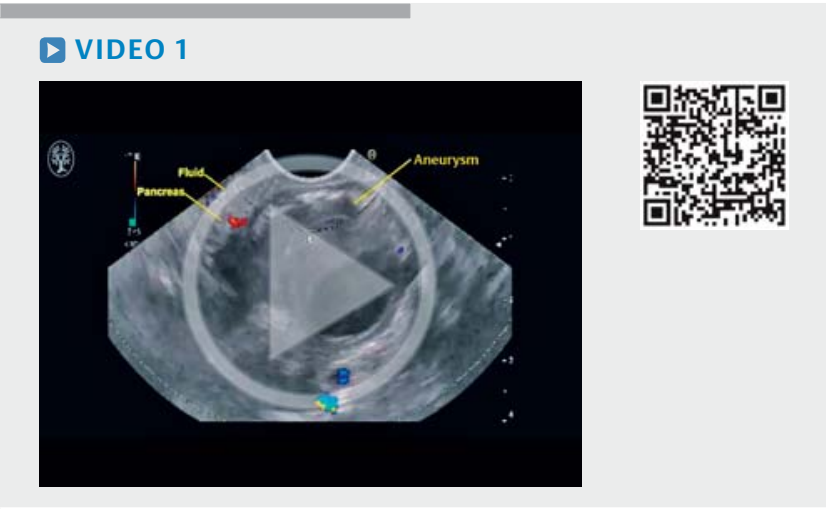

- Video 1: Endoscopic ultrasound (EUS) from the stomach showed a cystic lesion in the body of the pancreas, with a thick outer hypoechoic wall and an inner anechoic area, giving it a characteristic appearance of a "donut." EUS from the duodenum revealed edematous pancreatic parenchyma with peripancreatic fluid collection, consistent with an acute attack of pancreatitis. 
(• Fig.1c), consistent with an acute attack of pancreatitis. On color-flow and Doppler ultrasound imaging, vascular flow was seen in the central anechoic area ( $\triangleright$ Fig.1 d, $\triangleright$ Video 1 ), which confirmed the vascular nature of the lesion. EUS fine-needle aspiration (FNA) was not performed in view of the possibility of an aneurysm and the presence of acute inflammation. The patient underwent $\mathrm{CT}$ (abdomen), which revealed a $30 \times 26 \mathrm{~mm}$ splenic artery pseudoaneurysm. The aneurysm was successfully embolized and, at 6-month follow-up, he remained asymptomatic.

The most common abdominal visceral vessel affected by aneurysmal disease is the splenic artery, which has been attributed to acquired derangements of the vessel wall over time [1]. The incidence of pseudoaneurysm in chronic pancreatitis is about $6 \%-9 \%$ [2]. Ultrasound and CT are commonly used to evaluate vascular aneurysms. In some of these investigations, aneurysms can masquerade as pancreatic cystic lesions. In such cases, EUS can help in the final diagnosis. The aneurysms have a characteristic donutlike appearance at EUS: a thick outer wall with a central anechoic area. In a study by Varadarajulu et al., 4 out of 413 pancreatic cystic lesions were found to be aneurysms [3]. Awareness of aneurysm is necessary, as inadvertent FNA during EUS may lead to serious complications.

Endoscopy_UCTN_Code_CCL_1AF_2AG_3AD

\section{Competing interests}

None

The Authors

Malay Sharma ${ }^{1}$, Piyush Somani ${ }^{1}$, Maryam Al Khatry ${ }^{2}$, Amol Patil ${ }^{1}$

1 Department of Gastroenterology, Jaswant Rai Speciality Hospital, Meerut, India

2 Department of Gastroenterology, Obaidulla Hospital, Ras Al Khaimah, United Arab Emirates
Corresponding author

\section{Malay Sharma, MD}

Department of Gastroenterology, Jaswant Rai Speciality Hospital, Saket, Meerut, PIN-250 001, Uttar Pradesh, India,

Fax: +91-121-2657154,

sharmamalay@hotmail.com

\section{References}

[1] Trastek VF, Pairolero PC, Joyce JW et al. Splenic artery aneurysms. Surgery 1982; 91 : 694-699

[2] Fukatsu K, Ueda K, Maeda H et al. A case of chronic pancreatitis in which endoscopic ultrasonography was effective in the diagnosis of a pseudoaneurysm. World J Gastrointest Endosc 2012; 16: 335 - 338

[3] Varadarajulu S, Eloubeidi MA. Diagnosis of an aneurysm masquerading as pancreaticcyst lesion at EUS. Gastrointest Endosc 2007; 65: $721-725$

\section{Bibliography}

DOI http://dx.doi.org/10.1055/s-0042-120999 Endoscopy 2017; 49: E25-E26

(c) Georg Thieme Verlag KG

Stuttgart $\cdot$ New York

ISSN 0013-726X 